\title{
Reflexiones teórico-metodológicas para el abordaje de las personas adultas mayores en situación de vulnerabilidad: de la educación social a la pedagogía social, un estudio en la provincia de Alajuela, Costa Rica
}

Theoretical-Methodological Reflections for the Approach to Older Adults in Vulnerable Situations: From Social Education to Social Pedagogy, a Study in the Province of Alajuela, Costa Rica

Isabel Badilla Zamora

Universidad Nacional Heredia, Costa Rica isabel.badilla.zamora@una.cr

(D) https://orcid.org/0000-0002-6211-0470

Rafael Esteban Jiménez Corrales Universidad Nacional Heredia, Costa Rica rafael.jimenez.corrales@una.cr iD https://orcid.org/0000-0001-6004-6041

Heidy León Arce Universidad Nacional Heredia, Costa Rica heidy.leon.arce@una.crm

Anthia Ramírez García Universidad Nacional Heredia, Costa Rica. anthiarg@gmail.com 


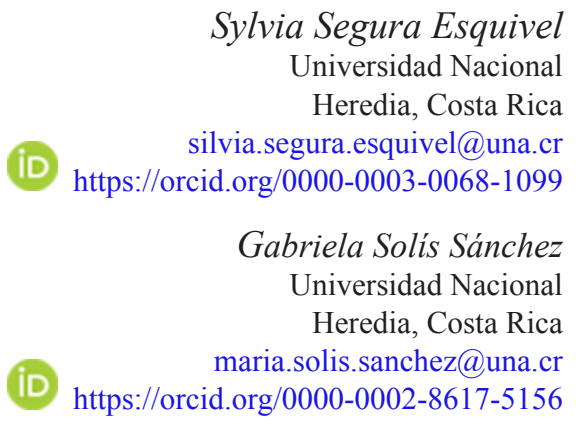

Recibido: 05/11/2020 • Aceptado:16/02/2021

Resumen. Este escrito sistematiza las reflexiones teórico-metodológicas para el abordaje de las personas adultas mayores en situación de vulnerabilidad y el proceso vivencial en la co-formación de una red de personas adultas mayores en la comunidad de Santa Rita de Alajuela, como parte del proyecto Pedagogía Social 2013-2018, de la Universidad Nacional (UNA), en el Centro de Investigación y Docencia en Educación (CIDE), en la División de Educación Básica (DEB). La metodología parte del paradigma naturalista, que considera que las personas adultas y adultas mayores participantes son constructoras de su pasado y actores sociales de su presente; ámbito que aborda la pedagogía social mediante la investigación-acción participativa. Una de las reflexiones a las que llega el equipo de investigación es que la población adulta mayor en dichas comunidades no solo requiere de atención física en salud, sino de espacios de recreación y de ocio, entendidos como herramientas para mejorar la salud integral, que contribuye en horas de alegría, de bienestar, de calidad de vida, lo que se verá reflejado en la salud emocional y física de las personas adultas mayores.

Palabras clave: persona adulta mayor, ocio, recreación, educación social, salud, pedagogía social, extensión, exclusión.

Abstract. This article systematizes theoretical and methodological considerations to care to vulnerable elders. It also systematizes the experiential process in jointly building a network of older adults in Santa Rita de Alajuela, as part of the 20132018 Social Pedagogy project being implemented by Universidad Nacional de Costa Rica (UNA) at Centro de Investigación y Docencia en Educación (CIDE) [Center for Education Teaching and Research] of UNA's Division of Basic Education (DEB). The methodology is based on the naturalistic paradigm and holds that participating adults and older adults are both builders of previous stages and social actors at current stages in their lives. This field is addressed by social pedagogy through participatory action research. A conclusion reached by the research team in the project is that the elderly population in this type of community requires not only care and healthcare but 
URL: http://www.revistas.una.ac.cr/index.php/dialogo/index

CORREO ELECTRÓNICO: universidadendialogo@una.cr

DOI: http://doi.org/10.15359/udre.11-2.5

also leisure and recreational spaces as tools to improve health thoroughly. This will result, in turn, in enjoyment, well-being, and quality of life and will be reflected in the emotional and physical health of the elderly.

Keywords: older person, leisure, recreation, social education, health, social pedagogy, outreach, exclusion.

\section{Introducción}

Desde el Proyecto de Pedagogía Social: UNA construcción para el aprendizaje de la organización de redes comunales socioeducativas surge la necesidad de conformar un grupo de personas adultas mayores en una comunidad caracterizada por su condición de pobreza, marginalidad, riesgo y vulnerabilidad social.

Los cimientos de esta red comunitaria de adultas y adultos mayores nacen a partir de las experiencias desarrolladas en el campo y de la iniciativa de personajes de la comunidad que apuestan a la construcción de lo que más tarde se convertiría en un puente de esperanza para cada persona participante del grupo.

A partir de las rutas metodológicas vividas en la comunidad de Santa Rita, este artículo pretende sistematizar la génesis y el desarrollo del grupo como red, también develar los principios sociopedagógicos que se diseñan en conjunto, para el mejoramiento de la calidad de vida de sus participantes.

Es importante señalar que, desde un inicio, la red grupal se autodenomina "Esperanza", la cual se encuentra ubicada en la comunidad de Santa Rita en la provincia de Alajuela en Costa Rica. Los principios sociopedagógicos que emergen de esta población se categorizan en dos grandes áreas: Principios en el hacer y principios en el ser.

Los primeros son las intencionalidades pedagógicas que están detrás de los momentos o las actividades que se desarrollan en cada encuentro, entre estos se pueden mencionar la espiritualidad, la movilización, la comensalidad, la actividad lúdica y el diálogo. Por otra parte, los principios pedagógicos del ser se definen como valores y principios morales indispensables para la convivencia y participación en el grupo. Entre estos destacan: el respeto, la tolerancia, la cortesía y el amor.

Las experiencias en y con el devenir de la red grupal han favorecido la construcción de diversas rutas metodológicas que pueden beneficiar u orientar la conformación de futuras agrupaciones de personas adultas y personas adultas mayores con características similares y en contextos de exclusión social. 


\section{Perspectiva teórica}

Siguiendo una lógica cualitativa, se establece una episteme dialéctica entre los hallazgos y las acciones dadas en el campo y la teoría, de esa forma se establecen las siguientes consideraciones teóricas.

\section{La situación de la persona adulta mayor en Costa Rica}

En las últimas décadas, la pirámide poblacional costarricense ha sufrido una alteración sumamente importante. La misma muestra un incremento sustancial de la población de personas adultas y personas adultas mayores. De esa forma, se indica que la esperanza de vida de la población costarricense ha alcanzado niveles sumamente elevados en comparación con el resto de la región latinoamericana.

Asimismo, se viene presentando un descenso notorio en la tasa de natalidad; para ello es importante destacar algunos datos tomados del Consejo Nacional de la Persona Adulta Mayor (Conapam, 2013) que indican que "según datos del Instituto Nacional de Estadísticas y Censos el porcentaje de la población adulta mayor con respecto a la población total para los siguientes años fue: en 1960 un 3,5\% en 1985 un 4,6\%, en el 2008 alcanzó un 6,2\% y para el 2035 se estima que llegará a un $15,9 \%$ ”. (p. 4)

Según la misma fuente usada por el Conapam, para el año 2013 la esperanza de vida al nacer ha crecido de forma sustancial, así se tiene que:

...En el año 1950 el promedio para hombres y mujeres fue de 57,7 años, 30 años después, es decir para el año 1980 subió a los 74,7 años y en 2010 pasó a 79,3 años, cifra que se ha mantenido hasta el 2013. En resumen, las personas adultas mayores cada vez son más y viven más. (p.4 y 5 ).

Es importante indicar que, en un país como Costa Rica, dada sus condiciones socioeconómicas, la edad tipificada según la normativa para considerarse persona adulta mayor es de 65 años o más. (Ley N. ${ }^{\circ} 7935$. p. 1). No obstante, tal como indica el Conapam (2013), la adultez mayor no es solamente un criterio etario, sino que involucra condiciones sociales que pueden adelantar o postergar dicha edad en términos de capacidades funcionales.

En un sentido más amplio, el proceso de envejecimiento está relacionado con aspectos psicosociales, económicos, culturales, educativos y familiares, la salud 
y la historia de vida, más que con el proceso de envejecimiento en sí. Lo señalado se refleja de forma clara y amplia en el análisis de datos del presente artículo.

\section{Pedagogía social y educación social}

Las acciones sobre las que se realiza esta experiencia tienen como soporte teórico fundamental a la pedagogía social, la cual es considerada como una ciencia social que estudia y analiza los hechos educativos, pero enfatizando en aquellos que se generan en la vida cotidiana de las personas. Es decir, dicha ciencia tiende a actuar en espacios educativos abiertos y no escolarizados, aunque no siempre renuncia a estos.

Como bien lo indica Pérez (2004), "de esa forma y siguiendo a la autora citada, la Pedagogía Social estudia la Educación Social o acciones educativas sociales que acontecen es espacios de menor nivel de escolarización" (citada por Senra y Vallés, 2010, pp. 184-185).

Consecuentemente, la pedagogía social no solo se ocupa de los procesos educativos en lugares y contextos relacionados con la educación, sino que atiende a los problemas de la vida cotidiana de las poblaciones. Por tanto, la realidad de la educación social no queda limitada a lugares de aprendizajes legitimados (como los centros de enseñanza organizados) y la familia, sino que busca también sus manifestaciones dentro de la misma sociedad.

Asimismo, la pedagogía social parte de la lógica de procesos constantes de socialización de los seres humanos, los cuales se vinculan con el bienestar de las personas, es decir, la socialización como meta de formación, la cual implica el mejoramiento individual y social de los seres humanos.

De esta forma, la pedagogía social, en contraste con el concepto de pedagogía general, como lo entiende Flórez (2000, p. 123), comparte sus tres grandes niveles de concretización:

a. Nivel teórico formal

b. Nivel teórico de intermediación y recontextualización

c. Nivel de aplicación y observación y generalizaciones empíricas

Por otra parte, y parafraseando a Senra y Vallés (2010), la pedagogía social abarca y estudia procesos educativos que al menos cumplan con dos de los siguientes atributos: 
a. Que se dirijan específicamente al desarrollo de la sociabilidad de personas.

b. Que tengan como destinatarios específicos a personas o colectivos en situación de conflicto social.

c. Que se realicen en contextos o por medios educativos no formales o no escolarizados.

De ahí que la relevancia de la propuesta escrita muestra con los hallazgos estos atributos.

\section{Principios pedagógicos: punto de partida fundamental en la acción educativa social}

Los principios pedagógicos son "sentidos" que toda corriente pedagógica posee, ellos pueden variar, complementarse e incluso contraponerse. Siguiendo a Flórez (2000), la pedagogía como ciencia estudiosa de los hechos educativos parte de grandes principios que se explicitan en el nivel teórico formal y se articulan con el nivel curricular de los modelos pedagógicos y de acción o aplicación.

De esa forma, se presentan diez principios generales, los cuales se citan a continuación. Estos funcionan en la teoría y en la práctica como puntos a considerar en la acción y en el análisis educativo:

1. El afecto, se comprende como la relacionalidad entre la persona pedagoga y los grupos con que se relaciona.

2. La experiencia natural, la cual se vincula con los intereses, las necesidades y los talentos de los y las aprendientes en su realidad sociocultural.

3. El diseño del medio ambiente, el cual realiza la persona pedagoga desde una mirada analítica de la situacionalidad del grupo de aprendientes.

4. El desarrollo progresivo, se relaciona con la comprensión y conciencia de la humanización de las personas durante todo su ciclo vital.

5. La actividad, es la conciencia que asume la persona aprendiente de sus herramientas conceptuales y morales, sus procesos de reelaboración interna.

6. La individualización, se relaciona con la propuesta en la cual el ser humano es, además de individuo y sujeto social, un individuo único y particular, una persona. 
7. Antiautoritarismo, este principio responde a las relaciones entre pedagogos, aprendientes y entre estos y las visualiza de forma horizontal.

8. Actividad grupal, favorece la comunicación asertiva y el diálogo entre los y las aprendientes.

9. Actividad lúdica, promueve que el juego prefigura y prepara para la vida.

10. La persona facilitadora idónea, es aquella persona pedagoga que vincula la acción de esta, de manera positiva, mirándolo como un ser que ofrece multivariadas formas y rutas de aprendizaje. Flórez (2010) lo llama el "buen maestro".

Partiendo de lo anterior, los principios son el andamiaje para que todo proceso educativo formal pueda llevarse a cabo de una mejor manera, logrando así no solo el bienestar del grupo que comparte el aprendizaje, sino que el o la aprendiente es capaz de interactuar de una forma armoniosa con su medio y con quienes están a su alrededor. De ahí que toda propuesta pedagógica o ruta metodológica que tome como fundamento dichos principios se garantiza el apego a la afectividad y a la comprensión de lo que es una verdadera acción educativa.

\section{Exclusión e inequidad social}

La pedagogía social posee como meta de formación la justa y adecuada integración del ser humano a la dinámica social, la cual a su vez debe tener una lógica orientada a la calidad de vida. De aquí se deriva que la exclusión social y la inequidad social son condiciones antipedagógicas.

Estos dos conceptos están muy unidos entre sí. El primero de ellos, la exclusión, se genera tanto de forma individual o colectiva y se caracteriza por el acto de impedir a los grupos y seres humanos participar de forma activa en la sociedad, en la definición y el alcance de un proyecto de vida de calidad.

Por lo general, la exclusión implica varios planos en los cuales se impide obtener logros educativos, económicos, sanitarios, laborales y otros. En los procesos de exclusión no todas las personas tienen las oportunidades y los recursos necesarios para participar de forma plena en la vida económica, social y política y así disfrutar de una vida con condiciones que le ayuden a su proceso de humanización.

Según Senra y Vallés (2010), una sociedad inclusiva poseerá los mecanismos para garantizar el goce de los derechos humanos y de paso permitirá a los grupos humanos la ciudadanía activa de sus integrantes. 
Por su parte, la inequidad representa una diferencia entre grupos o personas, esto implica una desigualdad de oportunidades para acceder a los diferentes bienes y servicios. El estado de inequidad puede ser dado por la clase o el grupo social al que se pertenece, por la procedencia geográfica, por la etnia, por el género al que se adscribe una persona. Algunas personas autoras consideran que la inequidad se asocia con la desigualdad de condiciones entre grupos o personas dentro del entramado social.

De esa forma, se genera inequidad cuando las clases o los grupos sociales no logran adquirir los mismos bienes y servicios; así un grupo por ingreso económico logra disponer de mayores bienes y servicios que otros grupos, con ello se genera además un trato desigual. La exclusión y la inequidad generan a su vez que grupos o personas sean discriminadas por alguna condición, ya sea personal o social.

Para varias personas autoras la expresión "exclusión social" implica la multidimensionalidad del término, lo cual lo puede hacer difuso y se vincula con el modo de desarrollo social. Es decir, es un concepto que presenta una profunda relatividad en el tiempo y en el espacio. El marco de los derechos humanos es un valioso intento por lograr un acuerdo que supere las diferencias culturales y temporales (Jiménez, Luengo y Tabemer, 2009).

\section{Metodología: un camino que apenas inicia}

Para efectos del presente escrito, el equipo de extensionistas-investigadores/ as parte de un paradigma naturalista, que considera que las personas adultas y las personas adultas mayores participantes son constructoras de su pasado y actores y actrices sociales de su presente.

Ellos y ellas han elaborado y elaboran formas de relacionarse en la vida y con la vida, han creado y crean formas de actuar e interactuar con el tejido social del cual forman parte, ya que son capaces de compartir experiencias, vivencias y aprendizajes del mundo circundante y de darles sentido y significado, así como de re-interpretarlos.

Se comprende que el pasado de las personas adultas mayores es parte fundamental dentro de la investigación, ya que este le da significado al contexto actual en el que se desarrollan.

De esa forma, se intenta mirar la realidad de las personas adultas y las personas adultas mayores sin necesidad de utilizar instrumentos rígidos, sino de una manera más espontánea, abierta y, en sus propias palabras, "divertida", y 
evita que la persona se sienta presionada. Sin que por lo anterior se pierda la coherencia teórica y metodológica del estudio.

Asimismo, el proceso investigativo y de extensión se aborda desde un enfoque cualitativo, porque "(...) permite recuperar el contexto y la dimensión humana (...)" (Bedmar y Añaños, 2006, p. 145); de la misma forma, explora, comprende y lee las realidades con las que se trabaja desde la complejidad.

Dado que el proceso se desarrolla en un ámbito de pobreza y exclusión social y al basarse en la pedagogía social, el estudio se ve en la necesidad metodológica y ética de adscribirse a la investigación-acción participativa, ya que esta "se orienta hacia la transformación social de poblaciones marginales (...)" (Bedmar y Añaños, 2006, p. 148).

Esta a su vez es una forma de comprender y crear rutas metodológicas como prácticas para el cambio social, con el fin de mejorar las condiciones socioemocionales y los aprendizajes en la vida, entre otros factores dentro del contexto en el que se desarrollan las personas participantes.

Por lo anterior, la ruta metodológica se enmarca con mayor peso, desde la visión universitaria, en el área académica de la extensión, entendida como la interrelación y el accionar de equipos de personas investigadoras y de estudiantes, entre otros, de la Universidad Nacional, en y con las comunidades nacionales, específicamente la población adulta mayor de la comunidad de Santa Rita, Barrio San José de la provincia de Alajuela.

Dicha ruta es visualizada de una manera humana, creadora y crítica, la cual busca enfrentar las necesidades de un grupo de la sociedad, en este caso, la población adulta y adulta mayor, especialmente porque se encuentran en desventaja cultural, social y económica, lo que deriva en una inadecuada calidad de vida.

Es así como se identifican diversas necesidades para redimensionar sus situaciones y plasmar acciones específicas que mejoren sus condiciones, en las que puedan aprender de sus propias experiencias y de esta forma compartirlas y resignificarlas con "los otros y las otras", estableciendo así el vínculo universidad-sociedad.

Es por esta razón que el proyecto Pedagogía Social, desde el 2013 hasta la fecha (en su segunda etapa), ha logrado no solo implementar acciones pedagógicas en pro de la población adulta y adulta mayor de la comunidad de Santa Rita, mediante tertulias, encuentros y talleres, sino que se propone como meta iniciar de forma conjunta la conformación de una red-grupo estable y autosostenido con dicho conglomerado social. 
En mayo del 2016, en el salón comunal de Santa Rita, se convocó a la población adulta mayor para dar inicio a un proceso: conformar un grupo estable y permanente; en ese momento asisten veinte personas adultas y personas adultas mayores, de ellas cinco eran hombres y quince mujeres, con edades entre los cincuenta y tres años y los noventa y siete años; cabe señalar, como lo establece la legislación costarricense, que la persona adulta mayor es toda aquella persona mayor de sesenta y cinco años. Por situaciones sociales y económicas, muchas personas envejecen de forma prematura. La norma cede ante las realidades sociales e individuales.

Dicho proceso se desarrolla en cinco fases que surgen del 2013 al 2017, a saber:

\section{Fase: Tardes del recuerdo}

Durante el periodo 2013-2015, se desarrolló en la región oeste de la provincia de Alajuela el proyecto Pedagogía Social: UNA Pedagogía sin Paredes (2013), el cual se llevó a cabo en varias comunidades del área en alianza con la Caja Costarricense del Seguro Social (CCSS). En cada una de las comunidades se desarrolló una fuerte fase de diagnóstico situacional. En ella se determinó que la población adulta mayor era sumamente numerosa y no estaba siendo atendida de forma integral.

En la comunidad de Santa Rita se logra establecer como hallazgo "la ausencia de una red - grupo de adultos y adultas mayores"; a partir de ese proceso, se procede a ejecutar varios encuentros con dicho sector poblacional, los mismos consisten en brindarles espacios, orientados hacia la promoción del ocio y de la recreación acorde a sus necesidades. En estos espacios, se ejecutan diversas técnicas, fundamentalmente la observación participante y las entrevistas informales, cumpliendo tanto la función diagnóstica como la función educativa y pedagógica de permitirle a la persona adulta mayor ser escuchada y reconocida como el legítimo otro. Es importante resaltar que los mismos se caracterizaban por la alegría, el gozo y los juegos, que eran parte de la dinámica.

\section{Fase: Nace un nuevo grupo}

Para inicios del año 2016, en su segunda etapa, el proyecto Pedagogía Social, en conjunto con dos líderes comunales, se reunieron para conversar sobre las posibilidades de crear un grupo de personas adultas mayores. Para entonces, se requería convocar nuevamente a los adultos y las adultas de la comunidad, para proponerles la idea. 
Cabe mencionar que la propuesta emergió con la participación de los y las presentes. Algunos aspectos a tratar eran los siguientes: establecer un día de reunión a convenir, un horario viable y el nombre del grupo, al igual que las disposiciones que como actores y actrices se requerían para socializar de una forma armónica, principalmente en el marco de principios éticos y morales.

Entre las técnicas y los instrumentos de mayor aplicación en esta fase estaban las entrevistas basadas en sus narraciones de vida desde la niñez hasta la edad adulta y los procesos y las formas de asentamiento en la comunidad (¿Cómo llegaron a instalarse en Santa Rita?), e incluso, en el caso de algunos y algunas en el país, su origen extranjero, mayoritariamente nicaragüense.

También, se aplicó la ficha biográfica y la técnica gráfica (dibujo inducido) como parte de la recolección de la información.

\section{Fase: La conformación de las acciones pedagógicas}

Cada semana de mayo a diciembre se fortalecía "la ruta metodológica de acción pedagógica". Dicha ruta se caracteriza por el ingreso a la lógica del adulto y de la adulta mayor del casco urbano, con carencias económicas, sociales y de cuido en general. En dichas sesiones se aplicaron algunas técnicas e instrumentos, tales como los grupos focales y las tertulias grupales basadas en temas de interés; además, la celebración como forma de reencontrarse con fechas importantes en sus vidas.

Para este momento se agrega un elemento muy valioso y es el intercambio generacional, al asistir en muchas ocasiones al grupo de personas adultas mayores estudiantes de las carreras de Educación Especial y Pedagogía en I y II Ciclos, que con su acompañamiento generaban nuevas experiencias. Cabe indicar que en esta etapa se generan procesos de deserción de algunas personas participantes, los motivos de tales situaciones se explicitan más adelante.

$\mathrm{Al}$ avanzar en dicha fase se culmina con el cierre del año cronológico del 2016 y se establecen nuevas metas junto con el grupo participante, es importante señalar que una de las metas trazadas por el grupo es la movilización hacia espacios fuera de la comunidad, con el fin de incentivar el ocio y la recreación. Por otra parte, se planteó la necesidad de crear una actividad de cierre de año.

\section{Fase: Sistematización de la experiencia}

Como todo proceso académico, se elabora una sistematización desde la perspectiva del equipo de extensionistas-investigadores/as y se analiza la 
información recopilada mediante las técnicas y los instrumentos aplicados en la experiencia. Para ello, se sigue una secuencia cronológica, desde la génesis del grupo hasta la creación y explicitación de una línea metodológica para el trabajo participativo con adultos y adultas mayores; se genera un diálogo con el fundamento de la teoría.

Es importante mencionar que durante esta fase los procesos de reflexión del equipo extensionista-investigador fueron sumamente profundos. Finalmente, se presentan los hallazgos y la construcción de la metodología para el trabajo con dicha población, línea fundamental para el desarrollo de la pedagogía social.

\section{Hallazgos y acciones pedagógicas: un devenir del Grupo Esperanza Policonsulta y estadísticas en conflicto}

La población mundial ha envejecido con el paso de los años, dicha tendencia ha sido y es sumamente sostenida en la realidad costarricense, siendo uno de los cambios demográficos más sorprendentes de los últimos tiempos.

Desde esta perspectiva, el proyecto pretende hacer conciencia sobre lo que representa el envejecimiento de la población y la necesidad de dar respuesta a las consecuencias personales, sociales y políticas derivadas de ello.

Entre los hallazgos más importantes del proyecto Pedagogía Social: una Pedagogía sin Paredes (2013-2016), se destaca el tema de la poli-consulta de parte de las personas adultas mayores a los Ebais, dicho término es entendido por el equipo de atención como "la asistencia repetida y sin mediar problema físico alguno a los centros de salud pública que atienden a las comunidades".

A partir de estas manifestaciones, el grupo de profesionales que se inscriben dentro de la pedagogía social genera un gran interés por trabajar en las comunidades, tales como Pacto del Jocote y Santa Rita, zonas con características de vulnerabilidad y con altos índices de desatención a la persona adulta mayor.

De esta manera, el Proyecto Pedagogía Social (2016-2018), en su segunda etapa, busca aportar a las comunidades participantes una esperanza y una actitud de cambio y de innovación; por otra parte, pretende mejorar las realidades socioeducativas de las personas adultas mayores, lo cual es de vital importancia entre los objetivos que persigue el proyecto.

Además, se favorece la convivencia entre personas y grupos distintos, con el fin de promover la igualdad y la equiparación de oportunidades desde el 
contexto comunitario; es decir, asumir un compromiso y vincular instituciones, instancias, grupos y centros, entre otros, y unificar esfuerzos con el propósito de crear redes comunales socioeducativas en la comunidad de San Rita.

Siendo la franja etaria de personas adultas mayores un grupo sumamente significativo, se decidió profundizar en esta realidad y se encontró que el estado de vulnerabilidad de esta población en específico se agrava de manera alarmante, por ejemplo en cuanto a la carencia de espacios y momentos de recreación y a la falta de atención y salud integral, lo cual ha provocado un alto índice en la poli-consulta en los Ebais, que se refleja en enfermedades crónicas, como secuelas de una mala salud preventiva y una conciencia de autocuidado deficiente a lo largo de la vida (Rodríguez et al., 2011).

Para esta segunda fase del proyecto se determinó que es necesario, mediante diversas acciones en el trabajo diario con las personas adultas mayores, que se logre una mejor condición de vida, tanto física y social como recreativa y mental; de manera que estas personas logren sentirse útiles y productivas, para que el fenómeno de poli-consulta se vea disminuido.

\section{Encuentros y rutas hacia el gozo}

La participación de la persona adulta mayor en la comunidad es de vital importancia para lograr mejores condiciones de bienestar, tanto en el área de la salud como en el área social. Indudablemente, con la integración de esta en actividades de la comunidad de una forma más activa, la persona adulta mayor puede mejorar su salud física y mental.

Al respecto, Ham (2003) indica que los factores sociales y económicos repercuten directamente en el bienestar integral de las personas y en la esperanza de vida.

Abordar el tema de la vejez desde una perspectiva integral, considerando sus historias de vida e integrándolas como personas que forman parte de una sociedad, que merecen respeto, amor y comprensión, logrará una placentera experiencia de gozo y de una mejor calidad de vida.

Desde esta visión, la participación de las personas adultas mayores a lo largo de estos dos años (2016-2017) representa una vivencia colmada de gozo, de experiencias de vida identitarias, con respecto al valor que tienen cada una de las personas adultas mayores que nos han acompañado en esta valiosa experiencia de encuentros y rutas de gozo. 
Propiciar el bienestar, la satisfacción personal y la mejora de sus condiciones y estilos de vida, de ocio, de recreación, de tiempo libre, de salud, de diálogos; estos son ejes trasversales que a lo largo de estos dos años han sido parte de los encuentros y las experiencias realizadas en la comunidad de Santa Rita.

\section{Los antecedentes de la creación del grupo-red de personas adultas mayores en la comunidad de Santa Rita}

El Proyecto Pedagogía Social en su primera fase (2013-2015) fue elaborando acciones comunitarias con sentido pedagógico, las cuales apuntaban al mejoramiento de la calidad de vida y la salud de diferentes sectores de las comunidades.

Como se ha dicho, en Santa Rita uno de los grupos detectados es el de la adultez mayor, de esa forma se realizaron acciones que buscaban cambiar la situación dada. Estas se orientaron de la siguiente forma:

Las Tardes de Recuerdo: Una experiencia mágica cargada de recuerdo, de afectividad, de ilusión y disfrute, donde un grupo de setenta personas adultas mayores comparten con sus mejores vestidos una tarde bailable, recordando épocas pasadas, rememorando historias a través de la conversación con otra persona, y con el aroma y deleite de un delicioso café o una aguadulce. Una tarde donde además de hacer un ejercicio vital para el cuerpo, se regocijan adorando a través del canto a su dimensión espiritual, la cual es entendida por ellos y ellas como Dios.

Tardes adorables y de aprendizaje mutuo, en las que se ha tenido la oportunidad de compartir experiencias y comentarios de las personas adultas mayores: "Que tarde más hermosa", "tenía mucho tiempo de no salir de la casa y esta actividad me motivó a salir y a disfrutar, como lo hacía cuando era joven". Indudablemente, un encuentro que fomenta la comunicación, la amistad y el establecimiento de relaciones interpersonales ricas y variadas.

La participación de las personas adultas mayores en los encuentros desarrollados ha generado en ellas múltiples beneficios, como disminuir sentimientos de soledad, mejorar su autoestima y su estado de ánimo y aumentar su motivación; se sienten parte de un grupo, aceptadas, con el reconocimiento comunitario. Estas actividades les han permitido mejorar su salud física y mental, aumentar su creatividad y el desarrollo de habilidades diversas.

\section{Terapia física y movimiento recreativo}

Muchas de las enfermedades de las personas adultas mayores crean condiciones o limitaciones tanto en el área de la salud física como en su vida social. Es por 
ello que desde la visión integral que plantea el proyecto el tema de la vejez es visto desde una perspectiva física, social y cultural.

Durante estos años, el proyecto se ha visto beneficiado por el aporte de varias personas profesionales y de múltiples experiencias con personas de la comunidad, así como por el trabajo conjunto de una fisioterapeuta, quien se caracteriza por tener una identidad muy arraigada hacia la comunidad y es reconocida como una líder, por su visión humana, integral y espiritual. Esto ha permitido que tanto las personas extensionistas como las personas adultas mayores se beneficien a través de actividades de terapia física que promueven el cuidado de la salud cognitiva, física y emocional.

El aporte de la fisioterapia en las sesiones de trabajo busca la independencia funcional de las personas adultas mayores, que logren mantener el equilibrio, la flexibilidad y se les facilite la expresividad corporal; además, que puedan enfrentar activamente la cotidianidad y las tensiones propias de esta etapa de la vida adulta, así como del contexto.

\section{Del clamor a la esperanza: génesis y conformación del grupo-red de personas adultas mayores}

"Cuando creas que no hay esperanza, algo nos sostiene y no se olvida de nosotros"; esta frase resume la posición que tienen muchas de las personas que habitan en la comunidad en riesgo social de Santa Rita, la cual miran como la posibilidad del llamado y clamor a un "Dios".

Muchos y muchas líderes comunales sienten que su clamor ha sido escuchado y que no es tarde para remediar la situación que su comunidad enfrenta; para ellos y ellas sus oraciones han tenido respuesta y "las esperanzas no están totalmente perdidas si la Universidad Nacional nos acompaña", como dicen, para abrir caminos.

Una de las líderes comunales logró movilizarse y, anteponiendo su sentido social a la lógica individualista, tomó la decisión de apoyar a la población de personas adultas mayores y buscar en la Universidad Nacional, representada por el proyecto Pedagogía Social, el apoyo necesario. Dicha líder comunal, nacida y crecida en la comunidad, ha logrado mediante estrategias familiares y personales estudiar terapeuta física.

Una vez que la líder inició su encuentro con el proyecto de la universidad, comenzó el entrecruzamiento de ideas y surgió la búsqueda de personas que quisieran liderar el grupo. Nuestra terapista física Jessenia comentaba la 
necesidad del abordaje a la población adulta mayor por el gran abandono que percibía hacia esta.

Además, se unió a la idea otro habitante de la comunidad, don Mario, manifestando ser también alguien que siempre deseaba apoyar a su comunidad y que le encantaría poder brindar su tiempo a este grupo. Se ignoraron todas las voces de aquellas personas que querían desmotivar y que decían que todo estaba perdido, y se inició la respuesta al clamor de unos pocos y unas pocas.

Jessenia y Mario, en la primera reunión, junto con los y las representantes del proyecto definieron como importante llegar a ser personas respetuosas, tolerantes, aprender a disfrutar del sano placer de vivir, utilizando las normas de convivencia; es decir, los valores; dar lo mejor de sí mismo/a esforzándose al máximo, para así tener disciplina y trabajar en equipo, para motivar la sociabilidad de las personas adultas mayores, cultivar la amistad y el compañerismo y contribuir a mejorar la calidad de vida de las personas de la tercera edad.

De esa forma, convocaron de boca en boca a la apertura de un grupo de personas adultas mayores, el cual se reuniría en el Salón Comunal. Llegó el día esperado, la primera reunión, en donde observábamos el reloj y conforme pasaba el tiempo fueron llegando las personas invitadas.

Con motivación, iniciamos la reunión, en la cual manifestaron la necesidad de reglas o normas de convivencia. A continuación, se enumeran: tratar bien a las personas para que las personas nos traten bien, tener respeto, entre todos y todas tomar decisiones, ser una persona educada, pedir las cosas "por favor" y ayudarnos entre sí. Porque aseguran que quieren recibir respeto dentro del grupo, que exista tolerancia, amor, paciencia, sinceridad, humildad, unión e igualdad. El papel de los y las proyectistas ha sido orientar, establecer alianzas, construir en conjunto con las personas participantes y darle un sentido formativo a la acción.

\section{Ruptura con aroma a café}

En algún momento, y en todo grupo social, el conflicto hace su aparición; por esto se genera lo que se ha denominado "ruptura con aroma a café". Así, durante el espacio que se brinda para la "tertulia del café", surgió un comentario con la única intención de cuidar la salud de don Mario, en cuanto a que no tomara tanto café, ya que su taza era muy particular, más grande de lo normal.

El comentario hecho por la compañera Jessenia y secundado por una compañera del proyecto solo buscaba apoyar su propio beneficio. No obstante, don Mario 
transformó aquellas palabras, en palabras con distinta profundidad aún más allá de nuestra lógica.

$\mathrm{Su}$ ausentismo de la siguiente reunión de grupo nos hizo pensar que seguía mal de salud, y esa era la preocupación mayor, él había estado internado, por lo que Jessenia decidió llamarlo; su lógica respondía a una persona ofendida y ofuscada por haber recibido un maltrato e indicaba que él podía cuidarse por sí solo y que no volvería al grupo.

Aunque no era lo esperado, el momento fue el indicado para comprender que él quería ser más parte del grupo que coordinador; en diferentes ocasiones Jessenia le solicitaba su colaboración para las diferentes actividades, pero él disfrutaba de compartir con el grupo. Además, en algún momento su compañera Jessenia le indicó que primero se les servía a todas las personas adultas mayores y que por último a las personas coordinadoras; situaciones que no fueron aceptadas por don Mario. Es así como se da la ruptura con aroma a café.

La actitud de don Mario es propia de la lógica comunitaria en riesgo social de Santa Rita, la idea de alejarme cuando algún poder institucional me obliga, o me convoca, idea aprendida desde la niñez, poco entendida y trabajada por la educación formal.

\section{La consolidación del grupo de personas adultas mayores. Construyendo un puente de esperanza}

El Grupo de Personas Adultas Mayores Esperanza (nombre que se generó luego de un proceso de elección democrática) se reúne una vez por semana en el Salón Comunal de Santa Rita. El grupo tiene una rutina de trabajo establecida y organizada por las mismas personas participantes del grupoy realmenterepresenta un producto entre miradas populares y miradas académico-pedagógicas. Esta rutina se divide en cinco partes que se describirán seguidamente.

Dan inicio con la oración, esta actividad espiritual es dirigida de forma alterna por los y las integrantes del grupo, en este espacio expresan su gratitud, ponen en oración a personas de la comunidad y realizan peticiones relacionadas con necesidades personales.

Seguidamente, una terapeuta física dirige una sesión de ejercicios, la cual consiste en un calentamiento que incluye estiramiento de las extremidades superiores e inferiores, posteriormente realizan marchas y desplazamientos y concluyen con ejercicios de respiración. 
La tercera actividad que se realiza corresponde al desarrollo de habilidades sociales, tales como: trabajo cooperativo, comunicación asertiva y seguimiento de instrucciones, así como habilidades relacionadas con la motricidad fina; se realizan diversos proyectos manuales que implican recortar, ensartar y pintar, entre otras actividades.

Después del trabajo manual, limpian y organizan las mesas, para dar paso al periodo de la comensalidad, un espacio de encuentro y comunión donde comparten, además de los alimentos, un poco de la esencia de sí mismos/as. El espacio anterior y este son aprovechados por los y las proyectistas para evaluar el proceso educativo y el devenir del grupo-red.

Finalmente, antes de concluir el encuentro, participan de una actividad lúdica, la cual se desarrolla a través de la dinámica del bingo. En este espacio de ocio y recreación se organizan de la siguiente manera: una persona colaboradora del grupo se encarga de cantar el bingo y los premios se obtienen de la colaboración de las mismas personas participantes del grupo. De mutuo acuerdo, establecieron la regla de ceder el premio a otro compañero o a otra compañera después de haber ganado una primera vez.

Concluyen la reunión con la limpieza y organización, distribuyendo funciones para el aseo del Salón Comunal. Todo momento reseñado tiene una intencionalidad pedagógica y formativa, lo cual confiere solidez al enfoque desde una pedagogía social que busca la activación en la sociedad de las personas adultas como grupo-red.

\section{Construcción de acuerdos y formas de asociación dentro del grupo}

En los diferentes encuentros con el grupo de participantes fueron manifestando una serie de condiciones o puntos de convergencia que los articulaba como tales, un conjunto de ideales dentro de los cuales el grupo genera un medio ético en el cual puede mantener su cohesión y existencia, los mismos conforman principios básicos que presentan un correlato con la teoría pedagógica que sustenta la investigación.

Para efectos de este análisis se categorizan dichos principios en dos grandes grupos. Por un lado, se tienen los principios en el ser del grupo, los cuales son manifestaciones directas que enunciaron los y las participantes ante cuestionamientos puntuales que buscaron puntos de convergencia y acuerdos para que el espacio grupal fuera agradable, viable y gozoso.

Por otra parte, se presentan los principios en el hacer del grupo, estos son formas de comportamiento o acción que se desarrollan en el devenir que 
URL: http://www.revistas.una.ac.cr/index.php/dialogo/index

CORREO ELECTRÓNICO: universidadendialogo@una.cr

DOI: http://doi.org/10.15359/udre.11-2.5

semana a semana se ha ido estableciendo para la convivencia grupal, y que las personas investigadoras deducen de las fuentes en diálogo con la teoría.

\section{Los principios en el ser}

Los principios en el ser del grupo se presentan a continuación:

a. Respeto: Implica aceptación de la palabra de cada persona participante del grupo.

b. Tolerancia: Se refiere a la aceptación de cada persona que integra el grupo en su individualidad totalizante.

c. Educación: Entendida desde el marco de acciones de cortesía, tales como saludos, agradecimientos y palabras de motivación

d. Puntualidad: Se manifiesta como el estar en el momento justo, en el espacio justo, de manera que no perjudique los tiempos de cada integrante del grupo.

e. Paciencia: Establecida como la espera y el respeto a los ritmos de ejecución de tareas y formas de ser de cada persona integrante

f. Honestidad: Entendida por las personas participantes como el respeto a la propiedad privada, en los objetos propios y valorados por ellos y ellas

g. Sinceridad: Implica la capacidad de manifestar y manifestarse entre sí los sentimientos que tienen para con otras personas.

h. Amor: Comprendido como la valoración de la presencia del ser de cada integrante, apreciar a las otras personas y extrañar la ausencia.

i. Amabilidad: Se relaciona con el buen trato, con el respeto en las interacciones que demanda cada una de las acciones y tareas que desarrolla el grupo de adultos y adultas.

j. Humildad: Las personas participantes del grupo lo entienden como no sentirse superior a nadie. Verse como iguales, como personas con aciertos, virtudes y carencias.

k. Unión: Este principio se visualiza como el ser del grupo, presencias constantes, participaciones, un llevarse bien, que fortalece la identidad del grupo en cuanto a pertenecer a un conglomerado propio, reconocido y no ajeno, lo cual se sintetiza en el nombre que se le asigna: Grupo de Adultos Mayores Esperanza de Santa Rita de Alajuela. 
1. Cariño: Es mostrar formas de aceptación ante los logros de las demás personas de forma afectiva.

m. Igualdad: Las personas participantes lo consideran como mirarse en un mismo plano, evitar las jerarquías y aceptar que se comparte una condición etaria.

n. Compañerismo: Son formas de camaradería, darse bromas afectivas que no alteren la estabilidad emocional de las personas integrantes.

o. Fe: Las personas participantes consideran que sus vidas y la del grupo se encuentran bajo los designios de un ser todopoderoso, superior a ellos y ellas, el cual indica y dicta los momentos de placer y tristeza.

Los principios enunciados desde la palabra directa de los adultos y las adultas mayores constituyen para ellos y ellas los basamentos de la organización, de la red que van entretejiendo sus vidas (ver figura 1). Se trata de pilares que sostienen el nodo, que de acatarse y cumplirse garantizan la permanencia de la existencia del grupo al cual se han adscrito de forma voluntaria.

\section{Figura 1}

Red personas adultas mayores Esperanza

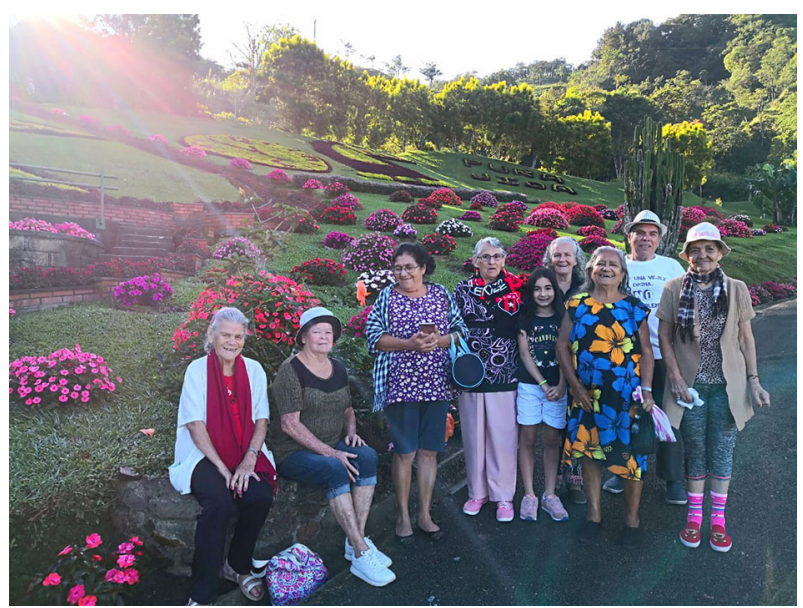

Nota. Del archivo del proyecto Pedagogía Social: UNA construcción para el aprendizaje de la organización de redes comunales socioeducativas (Badilla Zamora et al., 2016-2018). 
URL: http://www.revistas.una.ac.cr/index.php/dialogo/index

CORREO ELECTRÓNICO: universidadendialogo@una.cr

DOI: http://doi.org/10.15359/udre.11-2.5

\section{Los principios en el hacer}

Los principios en el hacer se estructuran en relación con los momentos que el grupo ha definido en su accionar, los mismos, como se señaló antes, emergen como categorías que definen las personas extensionistas en la vivencia y compartir con ellos y ellas y de los procesos de reflexión de los datos recolectados. Los mismos están distribuidos de la siguiente forma:

a. Momento de invocación a una existencia superior: Se evidencian tres principios: espiritualidad, gratitud y comunión. Son formas de religarse ante la totalidad de un ser que está más allá de la humanidad material, implican un profundo agradecimiento hacia la vida, la oportunidad de estar y ser reconocidos/as por una institución universitaria que es percibida como una instancia que desciende de las alturas de la academia y se planta en la realidad de la carencia, del dolor, del sufrimiento, de la exclusión, para convertirse en una luz de esperanza en las tinieblas de una edad que recuerda la finitud de sus seres (ver figura 2).

\section{Figura 2}

Momento de invocación a una existencia superior

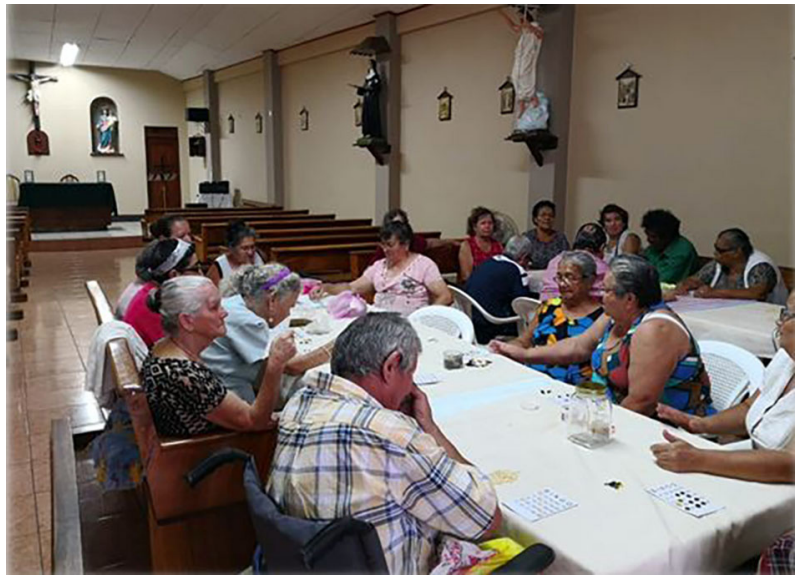

Nota. Del archivo del proyecto Pedagogía Social: UNA construcción para el aprendizaje de la organización de redes comunales socioeducativas (Badilla Zamora et al., 2016-2018). 
b. Momento del reconocimiento de la corporeidad: Se deducen los principios de movilización y autonomía. Es el espacio del reencuentro con los cuerpos de las personas adultas mayores. Cuerpos que al movilizarse se tornan en campos de gozo, el cual ha sido negado en gran parte de sus historias de vida y se ha visto en muchas oportunidades relegado a la producción, a la venta de la fuerza de trabajo, al brindar placer a otra persona que ha violentado esa corporeidad. Es, además, percatarse de que el cuerpo ha sido parte del devenir de la temporalidad, que no es el mismo. Es el acto de señalar "mi yo antes de la llegada del desgaste, del paso del tiempo en la exclusión y la inequidad social, cultural, económica que ha impedido una digna calidad de vida". No obstante, la corporeidad no solo recuerda privaciones y desventajas, sino que implica un derecho a no estar, a dejar fluir el movimiento en los otros y las otras y mirarlos y mirarlas con simpatía, con gozo y ternura (ver figura 3). A la vez, implica integrarme en el fluir del cuerpo, en sentir la música, en activar por medio del movimiento cuerpos que han permanecido silenciados por supuestos impedimentos sociales.

\section{Figura 3}

Momento del reconocimiento de la corporeidad
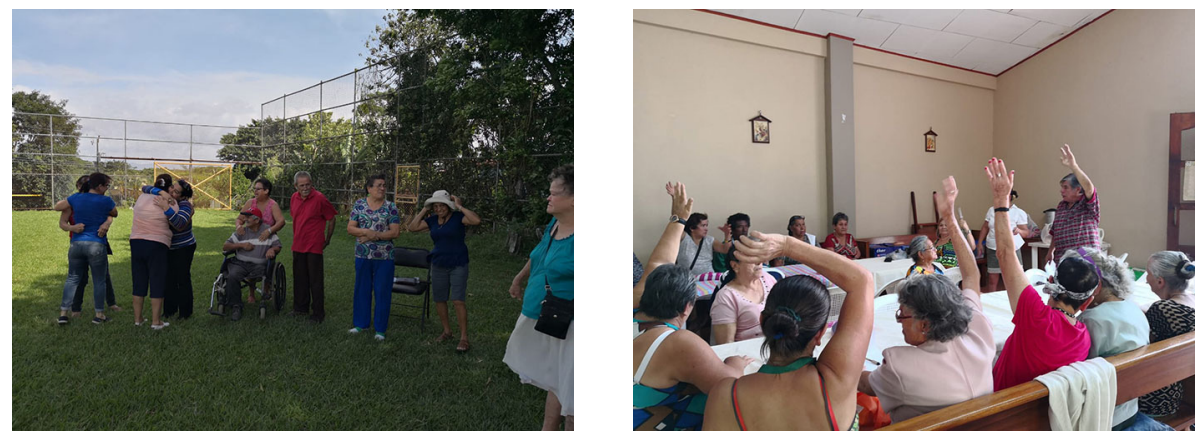

Nota. Del archivo del proyecto Pedagogía Social: UNA construcción para el aprendizaje de la organización de redes comunales socioeducativas (Badilla Zamora et al., 2016-2018).

c. Momento de la creación y externalidad de la creatividad: Se deducen los principios de expresividad verbal y plástica, comunicación asertiva, empatía, trabajo cooperativo, uso de la motora fina. Estos implican retomar y hacernos conscientes de la transformación de 
los elementos materiales, elaborando pequeñas obras que parten inicialmente de un modelaje, pero terminan siendo creaciones propias con elementos de mi medio ambiente, de mi existencia, que siendo carente se torna múltiple. En cada creación emerge el sello, la individualidad de cada integrante, ningún producto es idéntico al otro. Flores de plástico, mariposas, ojos en tela, son teñidos de los más insólitos colores, los cuales emergen de un acto creativo, de la propia visión de belleza que la vida ha generado en cada persona adulta mayor. En ese hacer las personas aprendientes se reconocen como seres comunitarios, que necesitan tanto del artefacto que me permite la transformación de la materia como de la ayuda de una mano amiga que le haga creador y creadora de algo nuevo (ver figura 4). En este acto emerge la necesidad y donación de una palabra que los y las inste a la conquista y al logro del proyecto que pasa del modelaje a la mente creativa que aún permanece en los cerebros abiertos al aprendizaje.

\section{Figura 4}

Momento de la creación y externalidad de la creatividad
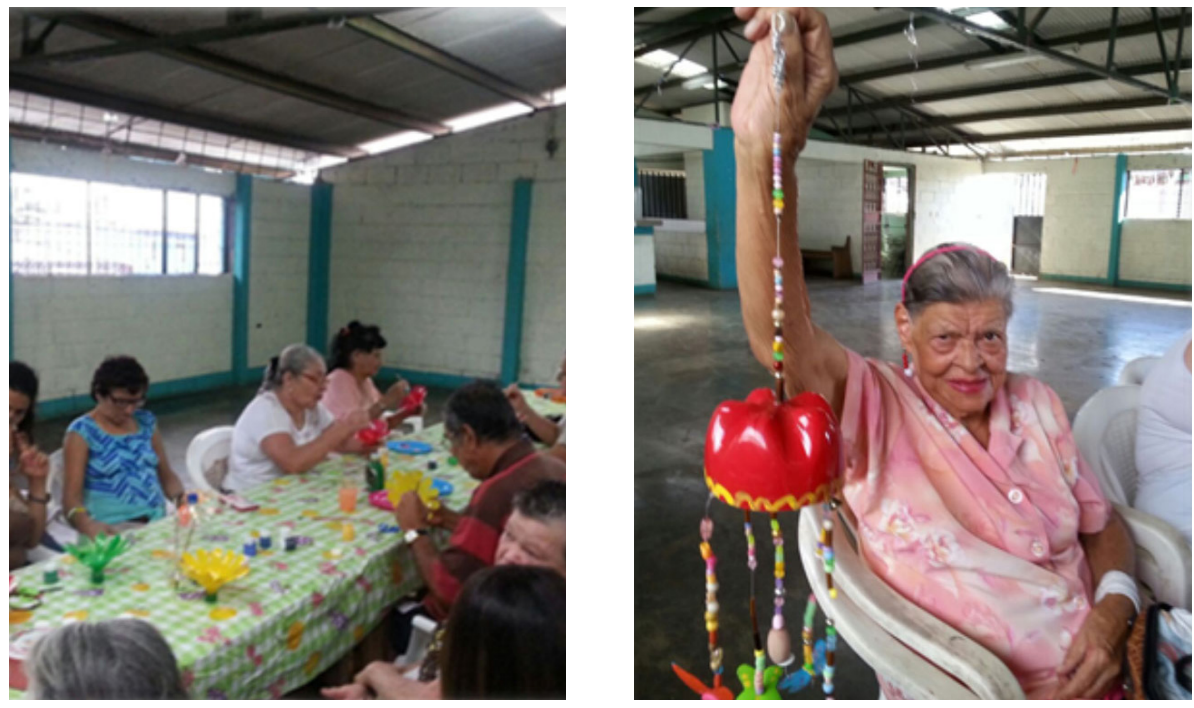

Nota. Del archivo del proyecto Pedagogía Social: UNA construcción para el aprendizaje de la organización de redes comunales socioeducativas (Badilla Zamora et al., 2016-2018). 
d. Momento de la comensalidad: En este se deducen los principios de gusto, satisfacción y comunión. Se plasma en la mesa, ahora las personas adultas son llamadas a la mesa, la cual es inclusiva, no privativa. Todo aquello que el pasado social ha castrado, sancionado y criticado se deja de lado para ser los y las comensales del altar de la reunión. Compartir los alimentos, si bien llena organismos carentes de elementos nutricionales, dado el contexto del grupo y su situación económica, llena seres carentes de afectividad, amor y reconocimiento. Se rompe la soledad en la que muchos y muchas viven y comen, para generar un acto compartido, un momento de satisfacción en que se transita de mi pan a nuestro pan (ver figura 5).

\section{Figura 5}

\section{Momento de la comensalidad}

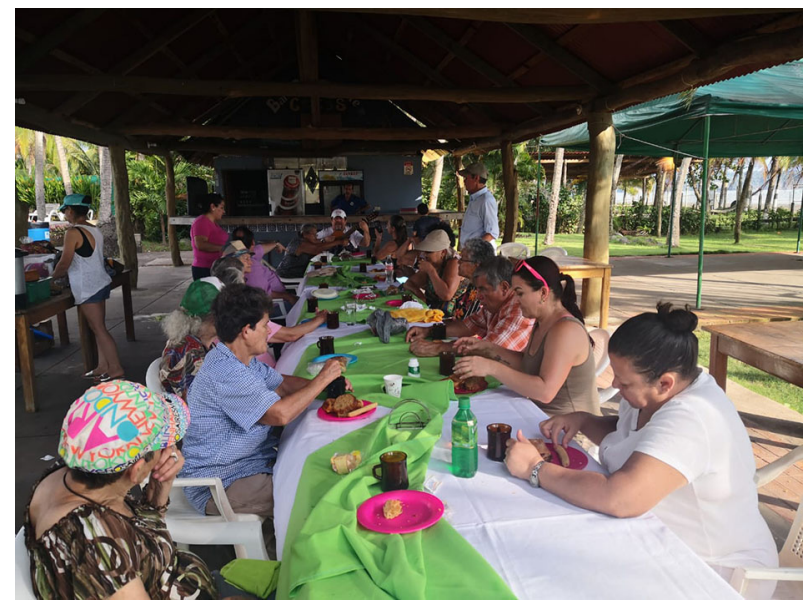

Nota. Del archivo del proyecto Pedagogía Social: UNA construcción para el aprendizaje de la organización de redes comunales socioeducativas (Badilla Zamora et al., 2016-2018).

e. Momento lúdico: En este momento se deducen los principios de alegría, incertidumbre, gozo y compartir con el otro y la otra que ha donado, ha regalado, aquello que a veces se arrebata de un rincón polvoriento, pero significativo del hogar y de mi carencia. El azar irrumpe en este momento como un aroma que transforma las vidas confinadas a la seguridad en la exclusión y la soledad. Aparece la expectativa, la oportunidad de la sorpresa que me eleva a la posibilidad 
de sentirme ganador y ganadora, jugador y jugadora. El azar no tiene elegidos ni elegidas, no distingue condición, simplemente emociona. Sucede como muchos de los grandes procesos universales y cósmicos que aún no son explicados porque quizá carecen de la racionalidad que ha impuesto un paradigma casi extinto de lo exacto, de la causaefecto. Produce por momentos la alegría de haber sido elegido y elegida, y cuando ese momento se torna reiterativo, la persona adulta mayor debe transitar de nuevo al reconocimiento de que existe otro y otra que no ha recibido la bendición del azar de las canicas que juegan y ruedan en la tómbola de la vida. Por otra parte, el juego evidencia la conciencia en el adulto y la adulta mayor de la incertidumbre de la vida. El estar al borde de alcanzar la meta de completar un cartón y no lograrlo trae la vivencia de que las cosas no son, sino que están abiertas al poder no ser (ver figura 6). Si bien este acto puede recordar fracasos de la vida, queda la esperanza de un nuevo encuentro en el cual podré salir victorioso o victoriosa.

\section{Figura 6}

\section{Momento lúdico}
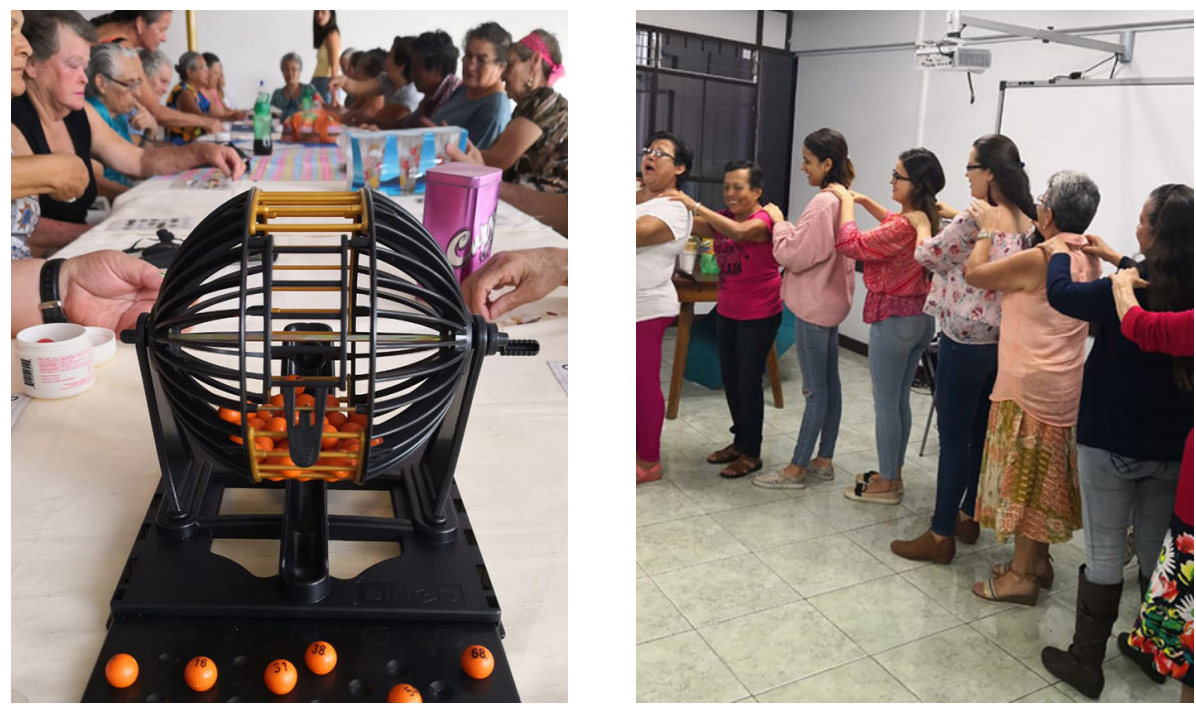

Nota. Del archivo del proyecto Pedagogía Social: UNA construcción para el aprendizaje de la organización de redes comunales socioeducativas Un estudio cruzado entre las comunidades de Santa Rita y Los Chiles de la provincia de Alajuela. (Badilla Zamora et al., 2016-2018). 


\section{Adultez mayor o envejecimiento prematuro}

El grupo de personas adultas mayores de la comunidad de Santa Rita posee características que validan los elementos teóricos aquí considerados, de esa forma la condición de adulto y adulta mayor por edad se relativiza al mirar la funcionalidad. Tal como señala el Conapam (2013): "En un sentido más amplio, el proceso de envejecimiento está relacionado con aspectos sicosociales, económicos, culturales, educativos y familiares, la salud y la historia de vida, que con el proceso de envejecimiento en sí” (p. 5).

Por tanto, las edades de las personas que conforman el grupo fluctúan entre los cincuenta y tres y los noventa y siete años. La condición etaria realmente se ve alterada. Las trayectorias vitales y las condiciones sociales, económicas $\mathrm{y}$ culturales hacen que las personas presenten un deterioro que las acerca súbitamente a una precoz adultez mayor.

Los principios del ser y del hacer del grupo de personas adultas mayores encuentran líneas comunes con grandes principios pedagógicos desarrollados por especialistas de la educación en general y de la educación social en particular.

\section{El principio de espiritualidad del grupo: referencia a los imaginarios de la comunidad}

En la comunidad de Santa Rita una las características de la dinámica social es poseer formas de comunicación y enfrentamiento a las diferencias de forma violenta. La institucionalidad nacional, pensada en y desde sectores medios y altos, utiliza otras formas de relacionalidad y de dimisión de conflictos. Lamentablemente, instituciones como las educativas no han profundizado o no se han percatado de estas conflictivas lógicas comunicativas.

Esas formas culturalmente aprendidas generan marginación e impiden formas de canalización de las demandas locales ante la institucionalidad. En los años ochenta y noventa, en Costa Rica surgió con gran fuerza el movimiento cristiano evangélico de naturaleza pentecostal. Existen diversas interpretaciones al respecto, ese movimiento parece inscribirse en las políticas ideológicas del periodo de Regan y Bush padre, que buscaban socavar los planteamientos de la teología de la liberación en América Latina, la cual había permeado tanto a sectores evangélicos como católicos.

Con el paso de los años, la ideología mencionada se profundiza y re-elabora, pero ya fuera de la Guerra Fría, lo cual ocasiona que el discurso se mezcle con 
ideas populares, generando nuevas formas de explicación de la pobreza, del conflicto y de la desigualdad en las comunidades.

Esa mixtura ideológica posee una fuerte presencia en Santa Rita, donde por encima de la conflictividad social y de la conflictividad cotidiana existe en los imaginarios un árbitro, un juez, una fuerza ordenadora de la vida: el Dios del Antiguo Testamento. Las formas organizativas pentecostales, la mayoría surgidas en el sur de los Estados Unidos, permean imaginarios, pero a la vez constituyen nuevas formas inéditas en comunidades como Santa Rita.

Esa mirada es compartida por gran parte de las personas adultas mayores, en la cual el Dios católico se mezcla con el Dios pentecostal, este segundo más libre, más "perdonador" y menos sacro en cuanto a su acceso.

En la vida de las personas adultas mayores, por encima de las fuerzas terrenas, operan las fuerzas divinas, por eso es necesario para muchos y muchas de sus habitantes "poner en las manos de Dios todas las áreas de la vida", la enseñanza indica que así nada se echará a perder.

Después de iniciar con la convocatoria de las personas adultas mayores y tener una guía de trabajo con el grupo discutida y aprobada en conjunto, se contempla iniciar con una oración a cargo de don Mario y continuar con un periodo de ejercicios físicos a cargo de Jessenia.

\section{La mirada de la pedagogía social en el accionar del grupo}

Por otra parte, el trabajo con el grupo de personas adultas mayores representa con toda claridad un proceso de educación social, el cual es atendido desde la pedagogía social, de esa forma se cumple con al menos dos de los atributos de dicha ciencia:

a. Se dirijan específicamente al desarrollo de la sociabilidad de sujetos.

b. Tengan como destinatarios específicos a individuos o colectivos en situación de conflicto social.

c. Se realicen en contextos o por medios educativos no formales o no escolarizados. (Senra y Vallés, 2010, pp. 184-185)

La presencia de los principios pedagógicos es fundamental en toda acción educativa, sea general o social, de esa manera muchos de los principios que Flórez (2000) establece se reafirman y aparecen en el ser y en el hacer del grupo en estudio, así se plasman algunos de ellos, tales como el afecto, la 
Revista Universidad en DiÁlogo • Vol. 11, N. 2, Julio-Diciembre, 2021 • 95-123

ISSN 2215-2849 • EISSN: 2215-4752

URL: http://www.revistas.una.ac.cr/index.php/dialogo/index CORREO ELECTRÓNICO: universidadendialogo@una.cr DOI: http://doi.org/10.15359/udre.11-2.5

individualización, la actividad grupal, la lúdica, el diseño del medio ambiente, el desarrollo progresivo y el cogobierno.

\section{Reflexiones finales}

Crear y desarrollar una red de personas adultas mayores en una comunidad en riesgo social en la provincia de Alajuela Costa Rica ha representado todo un reto y un aprendizaje. Los marcos teórico-metodológicos de la pedagogía general y de la pedagogía social han sido fundamentales para dicho proceso.

Uno de los factores del éxito de la red de personas adultas mayores con altos niveles de envejecimiento ha radicado en el proceso de no llevar la palabra teórica a la aplicación en la dinámica social, las ideas de construcción conjunta con los actores sociales, propia de los planteamientos de la investigaciónacción participativa, han sido cardinales en este proceso.

La lógica etnográfica para el trabajo con esta red no solo representa una fuente de develamiento de imaginarios sociales, sino que además ha permitido tomar esas construcciones comunales y darles un sentido pedagógico, sin violentar sus construcciones sociales.

En la comunidad de Santa Rita, los procesos educativos abiertos, los espacios para el ocio creativo y la recreación son sumamente limitados, la lógica de trabajar pedagógicamente la concretización de un espacio para personas adultas con envejecimiento prematuro y adultas mayores ha venido a dar una respuesta a un grupo social que posee un gran peso en la pirámide social costarricense y en esta comunidad.

La creación de formas alternativas y más inclusivas de comunicación y resolución de conflictos cotidianos representa grandes temas y aprendizajes vivenciados por este grupo de personas adultas mayores. Este punto ha quedado de lado por la institucionalidad oficial del Estado costarricense, sobre todo por la educación formal. No tener conciencia de ello limita la acción-comunitaria y perpetúa formas de comunicación violentas, cuya función cotidiana es efectiva, pero constituye a la larga uno de los elementos fundamentales para la manipulación política, la exclusión y la marginalidad social.

El equipo de investigadores concluye que la población adulta mayor en dichas comunidades no solo requiere atención física y en salud, sino espacios de recreación y de ocio, entendidos como herramientas para mejorar la salud integral. Esto contribuirá en horas de alegría, de bienestar y de calidad de vida, lo cual se verá reflejado en la salud emocional y física de las personas adultas mayores. 


\section{Referencias}

Asamblea Legislativa de Costa Rica. (15 de noviembre, 1999). Ley N. ${ }^{\circ} 7935$. Ley Integral para la Persona Adulta Mayor. La Gaceta N. ${ }^{\circ} 221$. San José, Costa Rica.

Bedman, M. y Añaños, F. (2006). Introducción a la pedagogía sociall educación social. Granada, España: Grupo Editorial Universitario.

Flórez, R. (2000). Pedagogía del conocimiento. McGraw Hill.

Ham, R. (2003). El envejecimiento en México: el siguiente reto de la transición demográfica. Porrúa y El Colegio de la Frontera Norte México.

Instituto Nacional de Estadística y Censo. (2011). X Censo Nacional de Población y VI de Vivienda: Resultados Generales. San José, Costa Rica.

Jiménez, M., Luengo, J. y Tabemer, J. (2009). Exclusión social y exclusión educativa como fracasos. Conceptos y líneas para su comprensión e investigación. Profesorado. Revista de Currículum y Formación de Profesorado, 13(3), 11-49. ISSN: 1138-414X. https://www.redalyc.org/ articulo.oa? id $=567 / 56712871002$

Rodríguez, E., Madrigal, D. y Alfaro, I. (2011). Análisis de la situación integral de salud 2011. Caja Costarricense de Seguro Social. Región Central Norte. Área de Salud Alajuela Oeste.

Senra, M. y Vallés, J. (2010). Compendio conceptual de educación social. Madrid: Ediciones Pirámide. 